\title{
Translation Corpus Source and Size in Bilingual Retrieval
}

\author{
Paul McNamee and James Mayfield \\ Human Language Technology Center of Excellence \\ Johns Hopkins University \\ Baltimore, MD 21218, USA \\ \{paul.mcnamee, james.mayfield\}@jhuapl.edu
}

\author{
Charles Nicholas \\ Dept. of Computer Science and Electrical Engineering \\ UMBC \\ Baltimore, MD 21250, USA \\ nicholas@umbc.edu
}

\begin{abstract}
This paper explores corpus-based bilingual retrieval where the translation corpora used vary by source and size. We find that the quality of translation alignments and the domain of the bitext are important. In some settings these factors are more critical than corpus size. We also show that judicious choice of tokenization can reduce the amount of bitext required to obtain good bilingual retrieval performance.
\end{abstract}

\section{Introduction}

Large parallel corpora are an increasingly available commodity. Such texts are the fuel of statistical machine translation systems and are used in applications such as cross-language information retrieval (CLIR). Several beliefs are commonly held regarding the relationship between parallel text quality and size for CLIR. It is thought that larger texts should be better, because the problems of data sparseness and untranslatable terms are reduced. Similarly, parallel text from a domain more closely related to a document collection should lead to better bilingual retrieval performance, again because better lexical translations are available.

We compared four sources of parallel text using CLEF document collections in eight languages (Braschler and Peters, 2004). English topic sets from 2000 to 2007 were used. Corpus-based translation of query terms was performed and documents were ranked using a statistical language model approach to retrieval (Ponte and Croft, 1998). Experiments were conducted using unlemmatized words and character 5-grams. No use was made of pretranslation query expansion or automated relevance feedback.

\section{Translation Corpora}

Information about the four parallel texts used in our experiments is provided in Table 1. We restricted our focus to Dutch (NL), English (EN), Finnish (FI), French (FR), German (DE), Italian (IT), Portuguese (PT), Spanish (ES), and Swedish (SV). These languages are covered by each parallel corpus.

\subsection{Bible}

The bible corpus is based on the 66 books in the Old and New Testaments. Alignments at the verse level were used; there are 31103 verses in the English text.

\subsection{JRC-Acquis v3}

This parallel text is based on EU laws comprising the Acquis Communautaire and translations are available in 22 languages. The English portion of the acquis data includes 1.2 million aligned passages containing over 32 million words, which is approximately 40 times larger than the Biblical text. Alignments were provided with the corpus and were produced by the Vanilla algorithm. ${ }^{1}$ The alignments are at roughly the sentence level, but only $85 \%$ correspond to a single sentence in both languages.

\subsection{Europarl v3}

The Europarl corpus was assembled to support experiments in statistical machine translation (Koehn, 2005). The documents consist of transcribed dialogue from the official proceedings of the European Parliament. We used the precomputed alignments that are provided with the corpus, and which are based on the algorithm by Gale and Church (1991). The alignments are believed to be of high quality.

\footnotetext{
${ }^{1}$ Available from http://nl.ijs.si/telri/vanilla/
} 


\begin{tabular}{|l|l|l|l|l|l|}
\hline Name & Words & Wrds/doc & Alignments & Genre & Source \\
\hline \hline bible & $785 \mathrm{k}$ & 25.3 & Near Perfect & Religious & http://unbound.biola.edu/ \\
\hline acquis & $32 \mathrm{M}$ & 26.3 & Good & EU law (1958 to 2006) & http://wt.jrc.it/lt/acquis/ \\
\hline europarl & $33 \mathrm{M}$ & 25.5 & Very Good & $\begin{array}{l}\text { Parliamentary oration } \\
\text { (1996 to 2006) }\end{array}$ & http://www.statmt.org/europarl/ \\
\hline ojeu & $84 \mathrm{M}$ & 34.5 & Fair & $\begin{array}{l}\text { Governmental affairs } \\
\text { (1998 to 2004) }\end{array}$ & $\begin{array}{l}\text { Derived from documents at } \\
\text { http://europea.eu.int/ }\end{array}$ \\
\hline
\end{tabular}

Table 1: Parallel texts used in experiments.

\subsection{Official Journal of the EU}

The Official Journal of the European Union covers a wide range of topics such as agriculture, trade, and foreign relations. We constructed this parallel corpus by downloading documents dating from January 1998 through April 2004 and converting the texts from Adobe's Portable Document Format (PDF) to ISO-8859-1 encoded text using pdftotext. The documents were segmented into pages and into paragraphs consisting of a small number of sentences (typically 1 to 3 ); however this process was complicated by the fact that many documents have outline or tabular formatting. Alignments were produced using Church's char_align software (1993).

Due to complexities of decoding the PDF, some of the accented characters were not extracted properly, but this is a problem mostly for the earlier material in the collection. In total about 85 million words of text per language was obtained, which is over twice the size of either the acquis or europarl collections.

\section{Translation}

Using the pairwise-aligned corpora described above, parallel indexes for each corpus were created using words and 5-grams. Query translation was accomplished as follows. For each query term $s$, source language documents from the aligned collection that contain $s$ are identified. If no document contains this term, then it is left untranslated. Each target language term $t$ appearing in the corresponding documents is scored:

$$
\operatorname{Score}(t)=\left(F_{l}(t)-F_{c}(t)\right) \times I D F(t)^{1.25}
$$

where $F_{l}$ and $F_{c}$ are relative document frequencies based on local subset of documents and the whole corpus. $I D F(t)$ is the inverse document frequency, or $\log _{2}\left(\frac{N}{d f(t)}\right)$. The candidate translation with the highest score replaced the original query term and the transformed query vector is used for retrieval against the target language collection.

This is a straightforward approach to query translation. More sophisticated methods have been proposed, including bidirectional translation (Wang and Oard, 2006) and use of more than one translation candidate per query term (Pirkola et al., 2003).

Subword translation, the direct translation of character n-grams, offers several advantages over translating words (McNamee and Mayfield, 2005). $\mathrm{N}$-grams provide morphological normalization, translations of multiword expressions are suggested by translation of word-spanning n-grams, and outof-vocabulary (OOV) words can be be partly translated with n-gram fragments. Additionally, there are few OOV n-grams, at least for $n=4$ and $n=5$.

\section{Experimental Results}

We describe two experiments. The first examines the efficacy of the different translation resources and the second measures the relationship between corpus size and retrieval effectiveness. English was the sole source language.

\subsection{Translation Resources}

First the relationship between translation source and bilingual retrieval effectiveness is studied. Table 2 reports mean average precision when word-based tokenization and translation was performed for each of the target collections. For comparison the corresponding performance using topics in the target language (mono) is also given. As expected, the smallest bitext, bible, performs the worst. Averaged across the eight languages only 39\% relative effectiveness is seen compared to monolingual performance. Reports advocating the use of religious texts for general purpose CLIR may have been overly optimistic (Chew et al., 2006). Both acquis and $\mathrm{eu}$ roparl are roughly 40 times larger in size than bible 


\begin{tabular}{|c|c|c|c|c|c|}
\hline Target & mono & bible & acquis & europarl & ojeu \\
\hline DE & 0.3303 & 0.1338 & 0.1802 & 0.2427 & 0.1937 \\
ES & 0.4396 & 0.1454 & 0.2583 & 0.3509 & 0.2786 \\
FI & 0.3406 & 0.1288 & 0.1286 & 0.2135 & 0.1636 \\
FR & 0.3638 & 0.1651 & 0.2508 & 0.2942 & 0.2600 \\
IT & 0.3749 & 0.1080 & 0.2365 & 0.2913 & 0.2405 \\
NL & 0.3813 & 0.1502 & 0.2474 & 0.2974 & 0.2484 \\
PT & 0.3162 & 0.1432 & 0.2009 & 0.2365 & 0.2157 \\
SV & 0.3387 & 0.1509 & 0.2111 & 0.2447 & 0.1861 \\
\hline Average & 0.3607 & 0.1407 & 0.2142 & 0.2714 & 0.2233 \\
& & $39.0 \%$ & $59.4 \%$ & $75.3 \%$ & $61.9 \%$ \\
\hline
\end{tabular}

Table 2: Mean average precision for word-based translation of English topics using different corpora.

\begin{tabular}{|c|c|c|c|c|c|}
\hline Target & mono & bible & acquis & europarl & ojeu \\
\hline DE & 0.4201 & 0.1921 & 0.2952 & 0.3519 & 0.3169 \\
ES & 0.4609 & 0.2295 & 0.3661 & 0.4294 & 0.3837 \\
FI & 0.5078 & 0.1886 & 0.3552 & 0.3744 & 0.3743 \\
FR & 0.3930 & 0.2203 & 0.3013 & 0.3523 & 0.3334 \\
IT & 0.3997 & 0.2110 & 0.2920 & 0.3395 & 0.3160 \\
NL & 0.4243 & 0.2132 & 0.3060 & 0.3603 & 0.3276 \\
PT & 0.3524 & 0.1892 & 0.2544 & 0.2931 & 0.2769 \\
SV & 0.4271 & 0.1653 & 0.3016 & 0.3203 & 0.2998 \\
\hline Average & 0.4232 & 0.2012 & 0.3090 & 0.3527 & 0.3286 \\
& & $47.5 \%$ & $73.0 \%$ & $83.3 \%$ & $77.6 \%$ \\
\hline
\end{tabular}

Table 3: Mean average precision using 5-gram translations of English topics using different corpora.

and both do significantly better; however europarl is clearly superior and achieves $75 \%$ of monolingual effectiveness. Though nearly twice the size, ojeu fails to outperform europarl and just barely beats acquis. Likely reasons for this include difficulties properly converting the ojeu data to text, problematic alignments, and the substantially greater length of the aligned passages.

The same observations can be seen from Table 3 where 5-grams were used for tokenization and translation instead of words. The level of performance with 5-grams is higher and these improvements are statistically significant with $p<0.01$ ( $t$-test). ${ }^{2} \mathrm{Av}$ eraged across the eight languages gains from $30 \%$ to $47 \%$ were seen using 5-grams, depending on the resource. As a translation resource europarl still outperforms the other sources in each of the eight languages and the relative ordering of \{europarl, ojeu, acquis, bible $\}$ is the same in both cases.

\footnotetext{
${ }^{2}$ Except in four cases: mono: In ES \& IT $p<0.05$; bible: 5 -grams were not significantly different than words in FI \& SV
}

\subsection{Size of Parallel Text}

To investigate how corpus size effects bilingual retrieval we subsampled europarl and used these smaller subcorpora for translation. The entire corpus is 33 million words in size, and samples of $1 \%$, $2 \%, 5 \%, 10 \%, 20 \%, 40 \%, 60 \%$, and $80 \%$ were made based on counting documents, which for europarl is equivalent to counting sentences. Samples were taken by processing the data in chronological order.

In Figure 1 (a-d) the effect of using larger parallel corpora is plotted for four languages. Mean average precision is on the vertical axes, and for visual effect the chart for each language pair uses the same scale. The general shape of the curves is to rise quickly as increasing subsets from $1 \%$ to $10 \%$ are used and to flatten as size increases further. Curves for the other four languages (not shown) are quite similar. The deceleration of improvement with increasing corpus size can be explained by Heap's Law. Similar results have been obtained in the few studies that have sought to quantify bilingual retrieval performance as a function of translation resource size $(\mathrm{Xu}$ and Weischedel, 2000; Demner-Fushman and Oard, 2003). In the higher complexity languages such as German and Finnish, n-grams appear to be gaining a slight improvement even when the entire corpus is used; vocabulary size is greater in those languages.

The data for the $0 \%$ condition were based on cognate matches for words and 'cognate n-grams' that require no translation. The figure reveals that even very small amounts of parallel text quickly improve performance. The $2 \%$ condition is roughly the size of bible, but is higher performing, likely due to a better domain match. ${ }^{3}$ Using a subsample of only $5 \%$ of available data from the highest performing translation resource, europarl, 5-grams outperformed plain words using any amount of bitext.

\section{Conclusion}

We examined issues in corpus-based bilingual retrieval, including the importance of parallel corpus selection and size, and the relative effectiveness of alternative tokenization methods. Size is not the only important factor in corpus-based bilingual re-

\footnotetext{
${ }^{3}$ For example, the Biblical text does not contain the words nuclear or energy and thus is greatly disadvantaged for a topic about nuclear power.
} 


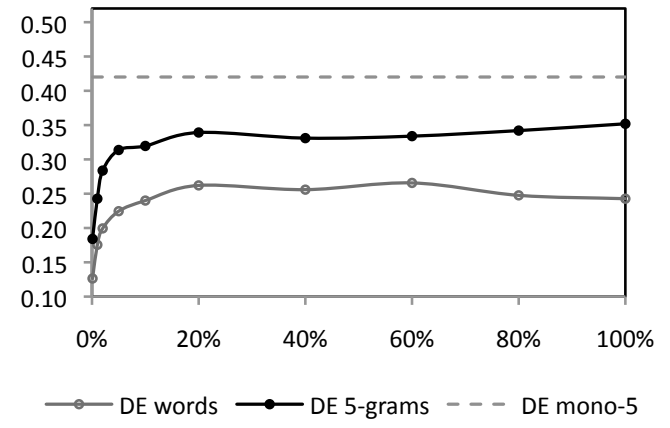

(a) German

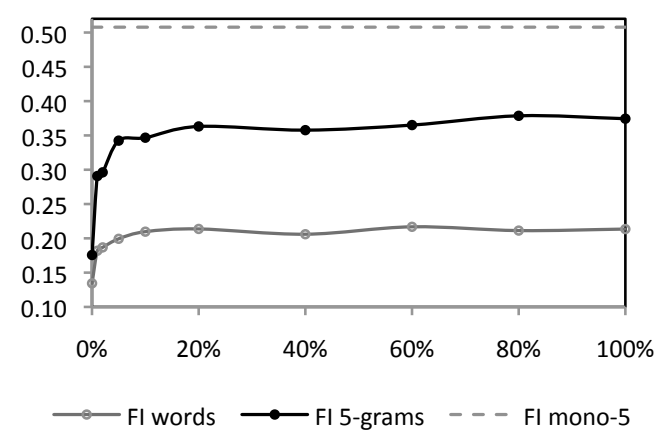

(c) Finnish

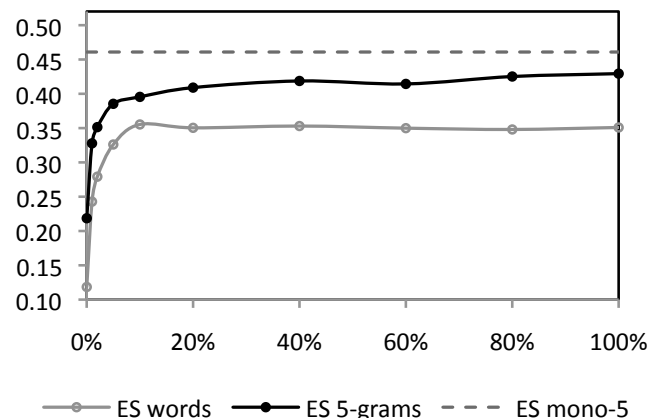

(b) Spanish

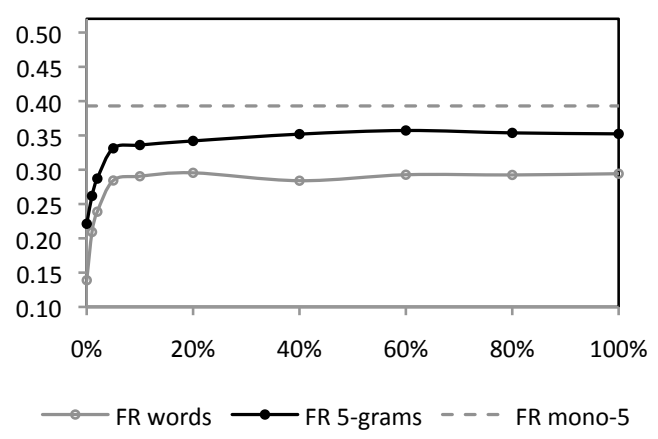

(d) French

Figure 1: Performance improvement with corpus growth.

trieval, the quality of alignments, compatibility in genre, and choice of tokenization are also important.

We found that character 5-gram tokenization outperforms words when used both for translation and document indexing. Large relative improvements (over 30\%) were observed with 5-grams, and when only limited parallel data is available for translation, n-grams are markedly more effective than words.

Future work could address some limitations of the present study by using bidirectional translation models, considering other language families and source languages other than English, and applying query expansion techniques.

\section{References}

Martin Braschler and Carol Peters. 2004. Crosslanguage evaluation forum: Objectives, results, achievements. Inf. Retr., 7(1-2):7-31.

P. A. Chew, S. J. Verzi, T. L. Bauer, and J. T. McClain. 2006. Evaluation of the Bible as a resource for cross-language information retrieval. In Workshop on Multilingual Language Resources and Interoperability, pages 68-74.

Kenneth Ward Church. 1993. Char_align: A program for aligning parallel texts at the character level. In Proceedings $A C L$, pages $1-8$.

Dina Demner-Fushman and Douglas W. Oard. 2003. The effect of bilingual term list size on dictionarybased cross-language information retrieval. In HICSS, pages $108-117$.

William A. Gale and Kenneth W. Church. 1991. A program for aligning sentences in bilingual corpora. In Proceedings ACL, pages 177-184.

Philipp Koehn. 2005. Europarl: A parallel corpus for statistical machine translation. In MT Summit.

Paul McNamee and James Mayfield. 2005. Translating pieces of words. In ACM SIGIR, pages 643-644.

Ari Pirkola, Deniz Puolamäki, and Kalervo Järvelin. 2003. Applying query structuring in cross-language retrieval. Inf. Process. Manage, 39(3):391-402.

Jay M. Ponte and W. Bruce Croft. 1998. A language modeling approach to information retrieval. In $A C M$ SIGIR, pages 275-281.

Jianqiang Wang and Douglas W. Oard. 2006. Combining bidirectional translation and synonymy for crosslanguage information retrieval. In $A C M$ SIGIR, pages 202-209.

Jinxi Xu and Ralph Weischedel. 2000. Cross-lingual information retrieval using hidden Markov models. In EMNLP, pages 85-103. 